\title{
There is no finite-variable equational axiomatization of representable relation algebras over weakly representable relation algebras. ArXiv version.
}

\author{
Jeremy F. Alm \\ Department of Mathematics \\ Illinois College \\ Jacksonville, IL 62650 \\ Robin Hirsch \\ Computer Science Department \\ University College London \\ r.hirsch@ucl.ac.uk \\ alm. academic@gmail .com \\ Roger D. Maddux \\ Department of Mathematics \\ Iowa State University \\ maddux@iastate.edu
}

December 17, 2015

\begin{abstract}
We prove that any equational basis that defines RRA over wRRA must contain infinitely many variables. The proof uses a construction of arbitrarily large finite weakly representable but not representable relation algebras whose "small" subalgebras are representable.
\end{abstract}

\section{Introduction}

Jónsson [7] axiomatized the class of lattices isomorphic to lattices of commuting equivalence relations. The operations of meet and join in such lattices are intersection and relational composition, respectively. Adding converse and the identity relation, Jónsson axiomatized the class of algebras isomorphic to algebras of binary relations with intersection, composition, converse, and identity as their operations. Applied to relation algebras, Jónsson's axioms yield a characterization of the the class of weakly representable relation algebras, i.e., the class of relation algebras isomorphic with respect to $0, \cdot, 1,,$, ; to algebras of binary relations with set-theoretic constants and operators $\emptyset, \cap, I d,{ }^{-1}, \mid$ ( see $[6$, Definition 5.14]). 
Jónsson asked whether his axioms (which were quasi-equations) could be replaced by equations. Pécsi [13] proved that they can. Jónsson proved there is a relation algebra that is not weakly representable, and asked whether there are weakly representable relation algebras that are not representable. Andréka [4] not only provided such examples, but showed that no finite number of first order conditions are enough to insure that a weakly representable algebra is representable.

Let RRA denote the class of representable relation algebras, and let wRRA denote the class of weakly representable relation algebras. Since wRRA is a variety, Andréka's result says that if $\Sigma$ is an equational basis that defines RRA over wRRA, that is, RRA $=w R R A \cap \operatorname{Mod}(\Sigma)$, then $\Sigma$ cannot be finite. In the present paper, we strengthen Andréka's result in Theorem 1:

Theorem 1. Suppose $\Sigma$ is a set of equations such that $\operatorname{RRA}=w \operatorname{RRA} \cap \operatorname{Mod}(\Sigma)$. Then the set of variables used by equations in $\Sigma$ is infinite.

This solves a problem from the first author's dissertation [1]. We solve another problem from [1] by exhibiting a non-representable relation algebra with a weak representation over a finite set. In addition, we reduce the size of the smallest known weakly representable but not representable relation algebra from $2^{366}$ (from [4]) to $2^{7}$ (see Corollary 13).

\section{Proof of Main Result}

Definition 2. A relation algebra $\mathfrak{A}=\left(A, 0,1,-,+, \cdot, 1^{\prime},{ }^{\smile}, ;\right)$ is a boolean algebra $(A, 0,1,-,+, \cdot$,$) together with an associative binary operation ; having identity$ element 1 ', i.e., $x=x ; 1$ ', and a unary operation ${ }^{`}$, satisfying additivity: $x ;(y+$ $z)=x ; y+x ; z,(x+y)^{\triangleleft}=\breve{x}+\breve{y}$, involution laws: $\breve{x}=x,(x ; y)^{\smile}=\breve{y} ; \breve{x}$ and the triangle law: $\breve{x} ; \bar{x} ; y \leq \bar{y}$. $\mathfrak{A}$ is symmetric if it satisfies $\breve{x}=x$, for all $x \in A$. $\mathfrak{A}$ is integral if 1 ' is an atom.

If $\mathfrak{A}$ is symmetric then $\mathfrak{A}$ is commutative (satisfies $x ; y=y ; x)$, by the involution laws. We only deal with finite symmetric (hence commutative) algebras.

Definition 3. Let $U$ be an equivalence relation over a set $D$. A representation $\theta$ of a relation algebra $\mathfrak{A}$ with unit $U$ over base $D$ is an injective map $\theta: \mathfrak{A} \rightarrow \mathcal{P}(U)$ sending each $a \in \mathfrak{A}$ to $a^{\theta}(\subseteq U)$, the image of a under $\theta$, that respects all the relation algebra operators and constants:

$$
\begin{aligned}
0^{\theta} & =\emptyset \\
1^{\theta} & =U, \\
\bar{x}^{\theta} & =U \backslash x^{\theta}=\left\{(u, v) \in U:(u, v) \notin x^{\theta}\right\}, \\
(x+y)^{\theta} & =x^{\theta} \cup y^{\theta}=\left\{(u, v) \in U:(u, v) \in x^{\theta} \vee(u, v) \in y^{\theta}\right\}, \\
(x \cdot y)^{\theta} & =x^{\theta} \cap y^{\theta}=\left\{(u, v) \in U:(u, v) \in x^{\theta} \wedge(u, v) \in y^{\theta}\right\}, \\
\left(1^{\prime}\right)^{\theta} & =\{(u, u): u \in D\}
\end{aligned}
$$




$$
\begin{aligned}
\breve{x}^{\theta} & =\left(x^{\theta}\right)^{-1}=\left\{(u, v) \in U:(v, u) \in x^{\theta}\right\}, \\
(x ; y)^{\theta} & =x^{\theta} \mid y^{\theta}=\left\{(u, v) \in U: \exists w \in D\left((u, w) \in x^{\theta} \wedge(w, v) \in y^{\theta}\right)\right\},
\end{aligned}
$$

$A$ weak representation is defined similarly, but need not respect union or complementation. RRA and $\mathrm{WRRA}$ are the classes of relation algebras that have representations and weak representations, respectively. As we mentioned earlier, they are both equational varieties. Given a representation (or a weak representation) $\theta$ over base $D$, any $x \in D$ and any $a \in \mathfrak{A}$ we write $\theta(a, x)$ for

$$
\left\{y \in D:(x, y) \in a^{\theta}\right\} .
$$

If $\theta$ is any weak representation of $\mathcal{A}$ whose unit is some equivalence relation $U$ over base $D$ then for any equivalence class $X$ of $U$ the map $\phi: \mathcal{A} \rightarrow \wp(X \times X)$

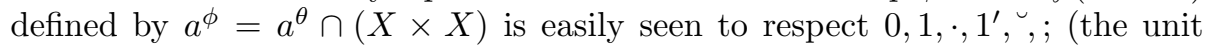
is now $X \times X$, the base is $X$ ). Further, if $\theta$ is a representation then $\phi$ also respects ${ }^{-},+$. If $\mathcal{A}$ is integral then it is easy to check, for non-zero $x \in \mathcal{A}$, that $x ; 1=1 ; x=1$ and this ensures that $\phi$ is injective. A representation (or weak representation) over base $D$ where the unit is $D \times D$ is called square. Since all the relation algebras considered in this paper are integral, if a representation (respectively weak representation) exists then a square (weak) representation also exists. When we refer to a (weak) representation over a set $D$ the unit will be assumed to be $D^{2}=D \times D$.

Lemma 4. If $\theta$ is a weak square representation of a relation algebra $\mathfrak{A}$ over a set $D$, then $\theta^{m}$ is a weak square representation of $\mathfrak{A}$ over $D^{m}$, where, for every $m \geq 1$ and every element $x$ of $\mathfrak{A}$,

$$
x^{\theta^{m}}=\left\{(u, v) \in D^{m} \times D^{m}: \forall i<m\left(\left(u_{i}, v_{i}\right) \in x^{\theta}\right)\right\} .
$$

Proof. The following calculations show that $\theta^{m}$ maps 1 , , , ; , and ' to the identity relation, intersection, relative product, and converse, respectively, just because $\theta$ does so.

$$
\begin{aligned}
\left(1^{\prime}\right)^{\theta^{m}} & =\left\{(u, v) \in\left(D^{m}\right)^{2}: \forall i<m\left(\left(u_{i}, v_{i}\right) \in\left(1^{\prime}\right)^{\theta}\right)\right\} \\
& =\left\{(u, v) \in\left(D^{m}\right)^{2}: \forall i<m\left(u_{i}=v_{i}\right)\right\} \\
& =\left\{(u, v) \in\left(D^{m}\right)^{2}: u=v\right\}, \\
(x \cdot y)^{\theta^{m}} & =\left\{(u, v) \in\left(D^{m}\right)^{2}: \forall i<m\left(\left(u_{i}, v_{i}\right) \in(x \cdot y)^{\theta}\right)\right\} \\
& =\left\{(u, v) \in\left(D^{m}\right)^{2}: \forall i<m\left(\left(u_{i}, v_{i}\right) \in x^{\theta} \cdot y^{\theta}\right)\right\} \\
& =\left\{(u, v) \in\left(D^{m}\right)^{2}: \forall i<m\left(\left(u_{i}, v_{i}\right) \in x^{\theta} \wedge\left(u_{i}, v_{i}\right) \in y^{\theta}\right)\right\} \\
& =\left\{(u, v) \in\left(D^{m}\right)^{2}: \forall i<m\left(\left(u_{i}, v_{i}\right) \in x^{\theta}\right) \wedge \forall i<m\left(\left(u_{i}, v_{i}\right) \in y^{\theta}\right)\right\} \\
& =x^{\theta^{m}} \cap y^{\theta^{m}}, \\
(x ; y)^{\theta^{m}} & =\left\{(u, v) \in\left(D^{m}\right)^{2}: \forall i<m\left(\left(u_{i}, v_{i}\right) \in(x ; y)^{\theta}\right)\right\} \\
& =\left\{(u, v) \in\left(D^{m}\right)^{2}: \forall i<m\left(\left(u_{i}, v_{i}\right) \in x^{\theta} ; y^{\theta}\right)\right\} \\
& =\left\{(u, v) \in\left(D^{m}\right)^{2}: \forall i<m \exists w_{i} \in\left(D^{m}\right)^{2}\left(\left(u_{i}, w_{i}\right) \in x^{\theta} \wedge\left(w_{i}, v_{i}\right) \in y^{\theta}\right)\right\}
\end{aligned}
$$




$$
\begin{aligned}
& =\left\{(u, v) \in\left(D^{m}\right)^{2}: \exists w \in\left(D^{m}\right)^{2}\left((u, w) \in x^{\theta^{m}} \wedge(w, v) \in y^{\theta^{m}}\right)\right\} \\
& =x^{\theta^{m}} \mid y^{\theta^{m}} \\
\breve{x}^{\theta^{m}} & =\left\{(u, v) \in\left(D^{m}\right)^{2}: \forall i<m\left(\left(u_{i}, v_{i}\right) \in \breve{x}^{\theta}\right)\right\} \\
& =\left\{(u, v) \in\left(D^{m}\right)^{2}: \forall i<m\left(\left(u_{i}, v_{i}\right) \in\left(x^{\theta}\right)^{-1}\right)\right\} \\
& =\left\{(u, v) \in\left(D^{m}\right)^{2}: \forall i<m\left(\left(v_{i}, u_{i}\right) \in x^{\theta}\right)\right\} \\
& =\left\{(u, v) \in\left(D^{m}\right)^{2}:(v, u) \in x^{\theta^{m}}\right\} \\
& =\left(x^{\theta^{m}}\right)^{-1}
\end{aligned}
$$

Definition 5. If $3 \leq p<\omega$ and $0 \leq n<\omega$, then $\mathfrak{L}(p, n)$ is the finite symmetric integral relation algebra with atoms $1^{\prime}, a_{0}, \ldots, a_{p}, t_{1}, \ldots, t_{n}$ such that, if $0 \leq$ $i, j \leq p, i \neq j, 1 \leq k, l \leq n$, and $k \neq l$, then

$$
\begin{aligned}
a_{i} ; a_{i} & =1^{\prime}+a_{i}, \\
a_{i} ; a_{j} & =0^{\prime} \cdot \overline{a_{i}+a_{j}}, \\
a_{i} ; t_{k} & =t_{1}+\cdots+t_{n}, \\
t_{k} ; t_{k} & =1^{\prime}+a_{0}+\cdots+a_{p}, \\
t_{k} ; t_{l} & =a_{0}+\cdots+a_{p} .
\end{aligned}
$$

Let $A=a_{0}+\cdots+a_{p}$ and $T=t_{1}+\cdots+t_{n}$. Whenever $0 \leq i, j \leq p$ and $i \neq j$, let $\mathfrak{L}^{i j}(p, n)$ be the subalgebra of $\mathfrak{L}(p, n)$ whose atoms are $1^{\prime}, a_{i}+a_{j}, a_{k}$ for $k \neq i, j$, $0 \leq k \leq p$, and $t_{1}, \ldots, t_{n}$ (when $n \geq 1$ ).

Note that $\mathfrak{L}(p, 0)$ has only the atoms $1, a_{0}, \ldots, a_{p}$, so $T=0, A=0$ ', and only the first two rules above govern relative multiplication of atoms in $\mathfrak{L}(p, 0)$. $\mathfrak{L}^{i j}(p, n)$ is indeed a subalgebra of $\mathfrak{L}(p, n)$ because all products involving the atom $a_{i}+a_{j}$ are joins of other atoms, as shown here:

$$
\begin{aligned}
\left(a_{i}+a_{j}\right) ;\left(a_{i}+a_{j}\right) & =1+A, \\
\left(a_{i}+a_{j}\right) ; a_{k} & =A \cdot \overline{a_{k}} \text { for } k \neq i, j, \\
\left(a_{i}+a_{j}\right) ; t_{l} & =T \text { for } 1 \leq l \leq n .
\end{aligned}
$$

In all of what follows, we assume $3 \leq p<\omega$ and $0 \leq n<\omega$.

Lemma 6. If $0 \leq i, j \leq p \leq q$ and $i \neq j$, then $\mathfrak{L}^{i j}(p, n)$ is isomorphic to a subalgebra of $\mathfrak{L}(q, n)$.

Proof. The map from the atoms of $\mathfrak{L}^{i j}(p, n)$ to $\mathfrak{L}(q, n)$ which maps $a_{i}+a_{j}$ to $a_{i}+a_{j}+\sum_{k=p+1}^{q} a_{k}$ and fixes all other atoms extends (using additivity) to an embedding of $\mathfrak{L}^{i j}(p, n)$ into $\mathfrak{L}(q, n)$.

Lemma 7. If $\theta$ is a representation of $\mathfrak{L}(p, n)$ over $D$ then

$$
p-1=\left|\theta\left(x, a_{i}\right)\right| \geq 2 n-1
$$

for all $x \in D$ and $0 \leq i \leq p$. 
Proof. Suppose that $\theta$ is a representation of $\mathfrak{L}(p, n)$ over $D$. Let $x \in D$. Then $(x, x) \in\left(1^{\prime}\right)^{\theta} \subseteq\left(a_{0} ; a_{0}\right)^{\theta}=a_{0}^{\theta} \mid a_{0}^{\theta}$ so there is some $x^{\prime} \in D$ such that $\left(x, x^{\prime}\right) \in a_{0}^{\theta}$. Now $a_{0} \leq a_{1} ; a_{i}$ for $i \in\{2, \ldots, p\}$, so there are distinct $y_{2}, \ldots, y_{p} \in \theta\left(x, a_{1}\right)$ such that $\left(y_{i}, x^{\prime}\right) \in a_{i}^{\theta}$ for $i \in\{2, \ldots, p\}$, hence $\theta\left(x, a_{1}\right) \supseteq\left\{y_{2}, \ldots, y_{p}\right\}$. Conversely, if $w \in \theta\left(x, a_{1}\right)$ then $\left(w, x^{\prime}\right) \in a_{1}^{\theta} \mid a_{0}^{\theta}=a_{2}^{\theta} \cup \cdots \cup a_{p}^{\theta}$, so there is some $j \in\{2, \ldots, p\}$ such that $\left(w, x^{\prime}\right) \in a_{j}^{\theta}$, hence $w=y_{j}$ because

$$
\left(w, y_{j}\right) \in\left(a_{1}^{\theta} \mid a_{1}^{\theta}\right) \cap\left(a_{j}^{\theta} \mid a_{j}^{\theta}\right)=\left(a_{1} ; a_{1} \cdot a_{j} ; a_{j}\right)^{\theta}=\left(1^{\prime}\right)^{\theta} .
$$

Therefore $\theta\left(x, a_{1}\right)=\left\{y_{2}, \ldots, y_{p}\right\}$ and $\left|\theta\left(x, a_{1}\right)\right|=p-1$. If $n=0$ or $n=1$ then $2 n-1 \leq p-1$ (since $p \geq 3$ ) and we are done, so assume $n \geq 2$.

Since $(x, x) \in t_{1}^{\theta} \mid t_{1}^{\theta}$, there is some $x^{\prime \prime} \in D$ such that $\left(x, x^{\prime \prime}\right) \in t_{1}^{\theta}$, as shown in the diagram below. Since $t_{1} \leq a_{1} ; t_{i}$ there are distinct $u_{1}, \ldots, u_{n} \in \theta\left(x, a_{1}\right)$ such that $\left(u_{i}, x^{\prime \prime}\right) \in t_{i}^{\theta}$, for $i \in\{1, \ldots, n\}$. Since $t_{i} \leq a_{1} ; t_{i}$ there are $v_{2}, \ldots, v_{n} \in$ $D$ such that $\left(u_{i}, v_{i}\right) \in a_{1}^{\theta}$ and $\left(v_{i}, x^{\prime \prime}\right) \in t_{i}^{\theta}$, for $i \in\{2, \ldots, n\}$. Note that $v_{2}, \ldots, v_{n} \in \theta\left(x, a_{1}\right)$ since $a_{1} ; a_{1}=a_{1}+1$, and that $u_{1}, \ldots, u_{n}, v_{2}, \ldots, v_{n}$ are distinct elements of $\theta\left(x, a_{1}\right)$, so $\left|\theta\left(x, a_{1}\right)\right| \geq 2 n-1$.

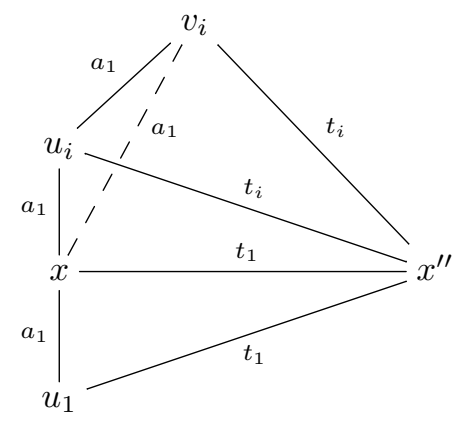

Corollary 8. If $2 n>p$ then $\mathfrak{L}(p, n) \notin \mathrm{RRA}$.

Lemma 9. If $p$ is a prime power then $\mathfrak{L}(p, 0)$ has a representation over a set of size $p^{2}$ and $\mathfrak{L}(p, 1)$ has a representation over a set of size $2 p^{2}$.

Proof. The first part was proved in [9, Theorem 1], along the following lines. Let $\mathbb{F}_{p}$ be the finite field of cardinality $p$. Let $D=\mathbb{F}_{p}^{2}$. $D$ is the affine plane with $p$ points on each line. Define some relations on $D$ as follows. If $0 \leq i<p$, $R_{i}$ is the set of pairs of distinct points that lie on lines with slope $i$, while $R_{p}$ is the set of pairs of distinct points that lie on a "vertical" line (with "infinite slope").

$$
\begin{aligned}
& R_{i}=\{(x, y): x, y \in D, y-x \in\{(j, i j): 0<j \in D\}\}, \text { for } 0 \leq i<p, \\
& R_{p}=\{(x, y): x, y \in D, y-x \in\{(0, j): 0<j \in D\}\} .
\end{aligned}
$$

Define a map $\phi: \mathfrak{L}(p, 0) \rightarrow \wp\left(D^{2}\right)$ by letting $\left(1^{\prime}\right)^{\phi}$ be the identity over $D$, $a_{i}^{\phi}=R_{i}$ (for $0 \leq i \leq p$ ) and extend $\phi$ by additivity to arbitrary elements of 
$\mathfrak{L}(p, 0)$. Then $\phi$ is a representation of $\mathfrak{L}(p, 0)$ on $D$. Let ${ }^{\prime}: D \rightarrow D^{\prime}$ be a bijection from $D$ to some disjoint set $D^{\prime}$ and let $\theta$ be defined on atoms of $\mathfrak{L}(p, 1)$ by

$$
\begin{aligned}
\left(1^{\prime}\right)^{\theta} & =\left\{(x, x): x \in D \cup D^{\prime}\right\}, \\
a_{i}^{\theta} & =R_{i} \cup\left\{\left(x^{\prime}, y^{\prime}\right):(x, y) \in R_{i}\right\}, \text { for } 0 \leq i \leq p, \\
t_{1}^{\theta} & =\left(D \times D^{\prime}\right) \cup\left(D^{\prime} \times D\right) .
\end{aligned}
$$

Extend $\theta$ by additivity to all of $\mathfrak{L}(p, 1)$. Then $\theta$ is a representation of $\mathfrak{L}(p, 1)$ over $D \cup D^{\prime}$.

Corollary 10. If $p$ is a prime power then $\mathfrak{L}(p, 0)$ has weak representations over finite sets of size $p^{2 m}$ for all $m \geq 1$.

Let $\theta$ be any weak representation of $\mathfrak{L}(p, 0)$ over a (possibly very large) finite base $D$. Again, let ' $: D \rightarrow D^{\prime}$ be a bijection from $D$ to some disjoint set $D^{\prime}$ and let $\theta^{\prime}$ be the weak representation of $\mathfrak{L}(p, 0)$ over $D^{\prime}$ defined by $\left(x^{\prime}, y^{\prime}\right) \in b^{\theta^{\prime}} \Longleftrightarrow$ $(x, y) \in b^{\theta}$ (for any $x, y \in D, b \in \mathfrak{L}(p, 0)$ ). Next we define a 'randomly labelled' $\mathfrak{L}(p, n)$ structure $\xi=\xi(\theta)$ over base $D \cup D^{\prime}$, as follows. Partition $D \times D^{\prime}$ into $n$ pieces $T_{1}, \cdots, T_{n}$ randomly, i.e., each pair $\left(x, y^{\prime}\right) \in D \times D^{\prime}$ is included in exactly one of the $T_{i}$ (some $1 \leq i \leq n$ ) with equal probabilities $\frac{1}{n}$ each, and the probabilities for distinct edges are independent. For $b \in \mathfrak{L}(p, n)$ let

$$
b^{\xi}=\left(b \cdot\left(A+1^{\prime}\right)\right)^{\theta} \cup\left(b \cdot\left(A+1^{\prime}\right)\right)^{\theta^{\prime}} \cup \bigcup_{t_{i} \leq b}\left(T_{i} \cup T_{i}^{-1}\right) .
$$

Lemma 11. Assume $\theta$ is a weak representation of $\mathfrak{L}(p, 0)$ over a base $D$. Let $d=|D|$ and $k \leq\left|\theta\left(a_{i}, x\right)\right|$ for all $x \in D, 0 \leq i \leq p$. Provided

$$
\begin{aligned}
& \left(\frac{n^{2}}{n^{2}-1}\right)^{d}>4 n^{2} d(d-1) \quad \text { and } \\
& \left(\frac{n}{n-1}\right)^{k}>4(p+1) n d^{2}
\end{aligned}
$$

the probability that the random structure $\xi$ is a weak representation of $\mathfrak{L}(p, n)$ is strictly positive.

Proof. For any distinct $x, y \in D$, any $z^{\prime} \in D^{\prime}$, and any $1 \leq i, j \leq n$ the probability that $\left(x, z^{\prime}\right) \in T_{i}$ and $\left(y, z^{\prime}\right) \in T_{j}$ is $\frac{1}{n^{2}}$. Hence, for any distinct $x, y \in D$ and any $1 \leq i, j \leq n$ the probability that there is no $z^{\prime} \in D^{\prime}$ such that $\left(x, z^{\prime}\right) \in T_{i}$ and $\left(y, z^{\prime}\right) \in T_{j}$ is $\left(\frac{n^{2}-1}{n^{2}}\right)^{d}$. Thus the probability that there is a distinct pair $x, y \in D$ and some $1 \leq i, j \leq n$ such that there is no $z^{\prime} \in D^{\prime}$ witnessing the product $t_{i} ; t_{j}$ is at most $d(d-1) n^{2}\left(\frac{n^{2}-1}{n^{2}}\right)^{d}$. Similarly, for $x \in D$, $y^{\prime} \in D^{\prime}, 0 \leq q \leq p$, and $1 \leq i \leq n$, the probability that there is no $z \in D$ such that $(x, z) \in a_{q}^{\rho}$ and $\left(z, y^{\prime}\right) \in T_{i}$ is $\left(\frac{n-1}{n}\right)^{\left|\theta\left(a_{q}, x\right)\right|}<\left(\frac{n-1}{n}\right)^{k}$. Hence the probability that $\xi$ fails to be a weak representation is less than

$$
2 d(d-1) n^{2}\left(\frac{n^{2}-1}{n^{2}}\right)^{d}+2(p+1) d^{2} n\left(\frac{n-1}{n}\right)^{k} .
$$


(2) and (3) ensure that this probability is strictly less than $\frac{1}{2}+\frac{1}{2}$, hence the probability that $\xi$ is a weak representation is strictly positive.

Lemma 11.5. If $x>(4 a)^{2}$ and $x>e^{\frac{b}{a}}$ then $x>a \log _{e}(x)+b$.

Proof. The first condition is equivalent to $\log _{e}(x)>2 \log _{e}(4 a)$. Recall that $e^{y}>y$ for all real $y$.

$$
e^{\log _{e}(x)-\log _{e}(4 a)}>\log _{e}(x)-\log _{e}(4 a)
$$

So

$$
\begin{aligned}
x & >4 a\left(\log _{e}(x)-\log _{e}(4 a)\right) \\
& >4 a \frac{\log _{e}(x)}{2} \quad(\text { by first condition) } \\
& =2 a \log _{e}(x) \\
& >a \log _{e}(x)+b \quad \text { (by second condition) }
\end{aligned}
$$

Theorem 12. If $p \geq 3$ is a prime power and $1 \leq n$, then $\mathfrak{L}(p, n)$ is weakly representable over arbitrarily large finite sets.

Proof. Let $\theta^{m}$ be the weak representation of $\mathfrak{L}(p, 0)$ given in (1) with base $D=\left(\mathbb{F}_{p}^{2}\right)^{m},|D|=p^{2 m}$, and note, for all $x \in D$ and all diversity atoms $a$ of $\mathfrak{L}(p, 0)$, that $|\theta(a, x)|=(p-1)^{m}$. Observe, in (2) and $(3)$, that $d=p^{2 m}$ and $k=(p-1)^{m}$ and that the left hand side of each inequality is governed by a double exponential function of $m$ whereas the right hand side is governed by only a single exponential function of $m$. Hence it is already clear that for sufficiently large $m$ both inequalities are satisfied. For such $m$, there is strictly positive probability that the random structure $\xi\left(\theta^{m}\right)$ is a weak representation on a base of size $2 p^{2 m}$ (Lemma 11 ), hence a weak representation $\xi$ exists within this probability space.

We claim that $(2)$ holds provided $m>\log _{p}\left(16 n^{2}\right)$ and $(3)$ holds provided $m>2 \log _{p-1}(24 n)$ and $m>\frac{1}{3} \log _{p-1}(4 n(p+1))$. Note, for $\alpha>1$

$$
\log _{e}\left(\frac{\alpha}{\alpha-1}\right)>\frac{1}{2 \alpha} \text {. }
$$

Now for the first part of the claim, suppose $m>\log _{p}\left(16 n^{2}\right)$. Then $p^{m}>16 n^{2}$ so $p^{2 m}>(4 \times 4 n)^{2}>2 n=e^{\frac{\log _{e}\left(4 n^{2}\right)}{2}}$. So by Lemma 11.5 with $a=4 n^{2}, b=$ $2 n^{2} \log _{e}\left(4 n^{2}\right)$,

$$
\begin{aligned}
p^{2 m}>\left(4 \times 4 n^{2}\right)^{2}, e^{\frac{\log \left(4 n^{2}\right)}{2}} & \Rightarrow p^{2 m}>4 n^{2} \log \left(p^{2 m}\right)+2 n^{2} \log \left(4 n^{2}\right) \\
& \Rightarrow \frac{p^{2 m}}{2 n^{2}}>\log \left(4 n^{2}\right)+2 \log \left(p^{2 m}\right)
\end{aligned}
$$




$$
\begin{aligned}
& \Rightarrow p^{2 m} \log \left(\frac{n^{2}}{n^{2}-1}\right)>\log \left(4 n^{2}\right)+2 \log \left(p^{2 m}\right) \text { by }(*) \\
& \Rightarrow\left(\frac{n^{2}}{n^{2}-1}\right)^{p^{2 m}}>4 n^{2} p^{2 m}\left(p^{2 m}-1\right)
\end{aligned}
$$

giving (2).

Now, for the second part of the claim, we show that (3) holds provided (i) $m>2 \log _{p-1}(24 n)$ and (ii) $m>\frac{1}{3} \log _{p-1}(4 n(p+1))$. Here's the proof. By (ii) we have $(p-1)^{m}>\sqrt[3]{4 n(p+1)}$ and by (i) we have $(p-1)^{m}>(24 n)^{2}$. So, by Lemma 11.5 with $a=6 n, b=2 n \log (4 n(p+1))$, we get $(p-1)^{m}>$ $6 n \log \left((p-1)^{m}\right)+2 n \log (4 n(p+1))$. Thus

$$
\begin{array}{rlr}
\frac{(p-1)^{m}}{2 n} & >\log (4 n(p+1))+3 \log \left((p-1)^{m}\right) & \\
& \geq \log (4 n(p+1))+2 \log \left(p^{m}\right) \quad(p \geq 3) .
\end{array}
$$

Hence (by $(*))$, we get $(p-1)^{m} \log \left(\frac{n}{n-1}\right)>\log (4 n(p+1))+\log \left(p^{2 m}\right)$ and so $\left(\frac{n}{n-1}\right)^{(p-1)^{m}}>4(p+1) n p^{2 m}$, yielding $(3)$.

For example, by Theorem 12 and Corollary 8, we have the smallest known weakly representable but not representable relation algebra:

Corollary 13. $\mathfrak{L}(3,2)$ is a non-representable relation algebra that is weakly representable over a finite set.

Theorem 14. If $p$ is a prime power and $p$ is large compared to $n \geq 1$, then $\mathfrak{L}(p, n)$ is representable over a finite set of size $2 p^{2}$.

Proof. The case $n=1$ is covered by Lemma 9, so assume $n \geq 2$. By Lemma 9, let $\theta$ be a representation of $\mathfrak{L}(p, 0)$ over a set $D$, where $|D|=p^{2}$ and $\left|\theta\left(u, a_{i}\right)\right|=$ $p-1$. If $p$ is sufficiently large compared to $n$ so that (2) and (3) hold then by Lemma 11 there is a strictly positive probability that the random structure $\xi(\theta)$ is a weak representation, hence a weak representation $\xi$ of this form exists. We show next that $p>16 n^{2}$ ensures (2) holds and $p>1+(48 n)^{2}$ ensures (3) holds. If $p>16 n^{2}$ then $p^{2}>2 n=e^{\frac{\log \left(4 n^{2}\right)}{2}}$. So by Lemma 11.5 with $a=4 n^{2}, b=2 n^{2} \log \left(4 n^{2}\right)$,

$$
\begin{aligned}
p^{2}>2 n^{2} \log \left(4 n^{2}\right)+4 n^{2} \log \left(p^{2}\right) & \Leftrightarrow \frac{p^{2}}{2 n^{2}}>\log \left(4 n^{2}\right)+2 \log \left(p^{2}\right) \\
& \Rightarrow p^{2} \log \left(\frac{n^{2}}{n^{2}-1}\right)>\log \left(4 n^{2}\right)+2 \log \left(p^{2}\right) \quad \text { by }(*) \\
& \Rightarrow\left(\frac{n^{2}}{n^{2}-1}\right)^{p^{2}}>4 n^{2} p^{2}\left(p^{2}-1\right)
\end{aligned}
$$

yielding (2).

Now suppose $p>(48 n)^{2}+1$. This is more than enough to get $p-1>e^{\frac{\log (4 n)}{6}}=$ 
$\sqrt[6]{4 n}$. So by Lemma 11.5 with $a=12 n, b=2 n \log (4 n)$ we get $(p-1)>$ $2 n \log (4 n)+12 n \log (p-1)$. Hence

$$
\begin{aligned}
\frac{p-1}{2 n}>\log (4 n)+6 \log (p-1) & \Rightarrow(p-1) \log \left(\frac{n}{n-1}\right)>\log (4 n)+4 \log (p+1) \quad p \geq 16 \\
& \Rightarrow\left(\frac{n}{n-1}\right)^{p-1}>4 n(p+1)^{5}>4 n(p+1)^{4} p
\end{aligned}
$$

yielding (3).

Since $\theta$ is a representation (not just a weak one) and since each edge from $\left(D \times D^{\prime}\right)$ is labelled by an atom below $T$, it follows that $\xi$ respects complement and is therefore a representation of $\mathfrak{L}(p, n)$.

Theorem 15. For every finite $\gamma$ there are $p$ and $n$ such that $\mathfrak{L}(p, n) \in$ wRRA $\backslash$ RRA and all the $\gamma$-generated subalegbras of $\mathfrak{L}(p, n)$ are representable over finite sets.

Proof. Pick any prime power $p$ such that $2^{\gamma}<p+1$ and pick $n>p / 2$. By Theorem $12 \mathfrak{L}(p, n)$ is weakly representable and by Corollary $8 \mathfrak{L}(p, n)$ is not representable. Let $\Gamma=\left\{g_{1}, \ldots, g_{\gamma}\right\} \subseteq \mathfrak{L}(p, n)$ be a set of $\gamma$ generators. Since $2^{\gamma}<p+1$ there are distinct $i, j \leq p$ such that for each $k$ either $a_{i}+a_{j}<g_{k}$ or $\left(a_{i}+a_{j}\right) \cdot g_{k}=0$. By Lemma 6 , the subalgebra of $\mathfrak{L}(p, 0)$ generated by the $\gamma$ elements $g_{k} \cdot A$ embeds into $\mathfrak{L}\left(p^{\prime}, 0\right)$ for all $p^{\prime} \geq p$. We may extend this embedding (and fix each $t_{i}$ ) to obtain an embedding of the $\Gamma$ generated subalgebra of $\mathfrak{L}(p, n)$ into $\mathfrak{L}\left(p^{\prime}, n\right)$. By Theorem 14 , if $p^{\prime}$ is sufficiently large compared to $n$ then $\mathfrak{L}\left(p^{\prime}, n\right)$ is representable over a finite set. Hence the subalgebra of $\mathfrak{L}(p, n)$ generated by $\Gamma$ is representable over a finite set.

Proof of Theorem 1. Suppose $\Sigma$ is a set of equations defining RRA over wRRA, i.e., $\mathrm{RRA}=\mathrm{wRRA} \cap \operatorname{Mod}(\Sigma)$. Also, suppose for contradiction that there is a finite $\gamma$ such that every equation $\varepsilon \in \Sigma$ contains only variables from $x_{1}, \ldots, x_{\gamma}$. Choose large odd prime power $p>2^{\gamma}-1$ and let $n=(p+1) / 2$.

Since $\mathfrak{L}(p, n)$ is not representable, but is weakly representable, there is some equation $\varepsilon \in \Sigma$ that is not valid in $\mathfrak{L}(p, n)$. By assumption, $\varepsilon$ contains at most $\gamma$ variables. Consider an assignment ' $:\left\{x_{1}, \ldots, x_{\gamma}\right\} \rightarrow \mathfrak{L}(p, n)$ to the variables, falsifying $\varepsilon$. Let $\operatorname{Sg}\left(x_{1}^{\prime}, \ldots, x_{\gamma}^{\prime}\right)$ be the subalgebra of $\mathfrak{L}(p, n)$ generated by $x_{1}^{\prime}, \ldots, x_{\gamma}^{\prime}$. Since each term using only variables $\left\{x_{1}, \ldots, x_{\gamma}\right\}$ evaluates under

' to the same thing in $\mathfrak{L}(n, k)$ as in $\operatorname{Sg}\left(x_{1}^{\prime}, \ldots, x_{\gamma}^{\prime}\right)$, this variable assignment falsifies $\varepsilon$ in $\operatorname{Sg}\left(x_{1}^{\prime}, \ldots, x_{\gamma}^{\prime}\right)$. But by Theorem $15, \operatorname{Sg}\left(x_{1}^{\prime}, \ldots, x_{\gamma}^{\prime}\right)$ is representable, yet it fails the equation $\varepsilon \in \Sigma$, contradicting the assumption RRA $=$ wRRA $\cap$ $\operatorname{Mod}(\Sigma)$

\section{Equational Complexity}

The following definition of equational complexity from [12] gives a sort of "measure" of non-finite-axiomatizability. 
Definition 16. The length of an equation is the total number of operation symbols and variables appearing in the equation. For example, the length of $(x+y) \cdot z=x \cdot z+y \cdot z$ is 12 .

For a variety $\mathrm{V}$ of finite signature, the equational complexity of $\mathrm{V}$ is defined to be a function $\beta_{\mathrm{V}}$ where for a positive integer $m, \beta_{\mathrm{V}}(m)$ is the least integer such that for any algebra $\mathcal{A}$ of the similarity class of $\mathrm{\vee}$ with $|\mathcal{A}| \leq m, \mathcal{A} \in \mathrm{\vee}$ iff $\mathcal{A}$ satisfies all equations true in $\mathrm{V}$ of length at most $\beta_{\mathrm{V}}(m)$. More generally, given two varieties $\mathrm{W} \subseteq \mathrm{V}$, the equational complexity of $\mathrm{W}$ over $\mathrm{V}$ is the function $\beta_{\mathrm{W} / \mathrm{v}}$ where for any positive integer $m, \beta_{\mathrm{W} / \mathrm{V}}(m)$ is the least integer such that for any algebra $\mathcal{A} \in \mathrm{V}$ with $|\mathcal{A}| \leq m, \mathcal{A} \in \mathrm{W}$ iff $\mathcal{A}$ satisfies all equations true in $\mathrm{W}$ of length at most $\beta_{\mathrm{W} / \mathrm{V}}(m)$.

In [2], a log-log lower bound was given for the equational complexity function for RRA. (See also [12].) Theorem 1 implies that the equational complexity function of RRA over wRRA must be unbounded; below, we give an explicit lower bound, also log-log.

Theorem 17. Let $\beta=\beta_{\mathrm{RRA} / \mathrm{wRRA}}$ be the equational complexity function of RRA over wRRA. Then for all $m \geq 2^{7}$,

$$
\beta(m)>\log _{2}\left(2 \log _{2}(m)-5\right)-\log _{2} 3 .
$$

Proof. From the proof of Theorem 15, we have that if $\mathfrak{A}$ is a $\gamma$-generated subalgebra of $\mathfrak{L}\left(p,\left\lceil\frac{p+1}{2}\right\rceil\right)$ with $\gamma<\log _{2}(p+1)$, then $\mathfrak{A}$ is representable, hence $\mathfrak{L}\left(p,\left\lceil\frac{p+1}{2}\right\rceil\right)$ satisfies all equations with $\gamma$ variables valid over representable algebras. Since $\mathfrak{L}\left(p,\left\lceil\frac{p+1}{2}\right\rceil\right)$ is not representable and $\left|\mathfrak{L}\left(p,\left\lceil\frac{p+1}{2}\right\rceil\right)\right|=2^{2+p+\left\lceil\frac{p+1}{2}\right\rceil}$, it follows that $\log _{2}(p+1) \leq \beta\left(2^{2+p+\left\lceil\frac{p+1}{2}\right\rceil}\right)=\beta\left(2^{\left\lceil\frac{3 p+5}{2}\right\rceil}\right)$. For any $m \geq 2^{7}$ (= $2^{\frac{3 \times 3+5}{2}}$ ) we can find $p \geq 3$ such that $2^{\left\lceil\frac{3 p+5}{2}\right\rceil} \leq m<2^{\left\lceil\frac{3 p+7}{2}\right\rceil} \leq 2^{\frac{3 p+8}{2}}$. Then

$$
\frac{2 \log _{2}(m)-8}{3}<p .
$$

Adding one and then taking logs of both sides of (4) yields

$$
\begin{aligned}
\log _{2}\left(\frac{2 \log _{2}(m)-5}{3}\right) & <\log _{2}(p+1) \\
& \leq \beta\left(2^{\left\lceil\frac{3 p+5}{2}\right\rceil}\right) \\
& \leq \beta(m),
\end{aligned}
$$

where the last line follows from monotonicity of $\beta$.

Therefore $\beta(m)>\log _{2}\left(2 \log _{2}(m)-5\right)-\log _{2} 3$ 


\section{Open Questions}

Naturally, it seems likely that any equational basis for wRRA contains infinitely many variables.

Problem 1. Does wRRA have a finite-variable equational basis?

The proof of Lemma 9, essentially due to Roger Lyndon, shows that $\mathfrak{L}(m, 0)$ is representable whenever there is an affine plane of order $m$. Furthermore, Lyndon proves the converse: if there is no affine plane of order $m$ then $\mathfrak{L}(m, 0)$ is not representable.

Problem 2. Is $\mathfrak{L}(m, 0)$ weakly representable for all finite $m \geq 3$ ?

If the answer to Problem 2 is "Yes", that would give a cleaner proof of the main result of the present paper. If the answer is "No", for infinitely many $m$, it would yield a negative answer to Problem 1.

Problem 3. Find a reasonable lower bound for the equational complexity function for wRRA.

A Monk algebra is an algebra derived from $\mathfrak{E}_{k+1}^{\{2,3\}}$ by splitting diversity atoms (see [5]). The algebras $\mathfrak{E}_{k+1}^{\{2,3\}}$ for $1 \leq k \leq 400$ were recently shown in [3] to be representable (except possibly for $k=8,13$ ). Splitting can destroy representability, however, as in the present paper.

Problem 4. Are all the Monk algebras weakly representable?

It is known [10] and follows from Theorem 15 that any equational theory defining RRA must use infinitely many variables, but now consider arbitrary first order theories.

Problem 5. Is there a first order theory (necessarily infinite) that defines RRA using only finitely many variables? If so, how many variables are needed?

Failing that:

Problem 6. Is there a first order theory (necessarily infinite) that defines RRA over wRRA using only finitely many variables?

\section{References}

[1] J. Alm. Weak representation theory in the calculus of relations. ProQuest LLC, Ann Arbor, MI. Thesis (Ph.D.), Iowa State University, 2006.

[2] J. Alm. On the equational complexity of RRA. Algebra Universalis, 68(3):321-324, 2012.

[3] J. Alm. and J. Manske. Sum-free cyclic multi-bases and constructions of Ramsey algebras. Discrete Applied Mathematics, 180:204-212, 2015. 
[4] H. Andréka. Weakly representable but not representable relation algebras. Algebra Universalis, 32(1):31-43, 1994.

[5] H. Andréka, R. D. Maddux, and I. Németi. Splitting in relation algebras. Proc. Amer. Math. Soc., 111(4):1085-1093, 1991.

[6] R. Hirsch and I. Hodkinson. Relation algebras by games, volume 147 of Studies in Logic and the Foundations of Mathematics. North-Holland Publishing Co., Amsterdam, 2002.

[7] B. Jónsson. Representation of modular lattices and of relation algebras. Trans. Amer. Math. Soc., 92:449-464, 1959.

[8] B. Jónsson, and A. Tarski. Boolean algebras with operators II. Amer. Journal of Math., 74:127-162, 1952.

[9] R. Lyndon. Relation algebras and projective geometries. Michigan Mathematics Journal, 8(1):22-28, 1961.

[10] B. Jónsson. The theory of binary relations. In Algebraic Logic, Colloq. Math. Soc. J. Bolyai, 54:245-292, North Holland, 1991.

[11] R. D. Maddux. Relation algebras, volume 150 of Studies in Logic and the Foundations of Mathematics. Elsevier B. V., Amsterdam, 2006.

[12] G. McNulty, Z. Székely, and R. Willard. Equational complexity of the finite algebra membership problem. Internat. J. Algebra Comput., 18(8):12831319, 2008.

[13] B. Pécsi. Weakly representable relation algebras form a variety. Algebra Universalis, 60(4):369-380, 2009. 